\title{
FACTORES RELACIONADOS CON LA ACTIVACIÓN LÍQUIDA DE MICROORGANISMOS DE MONTAÑA (MM)
}

\author{
Leida Castro-Barquero', José González-Acuña ${ }^{2}$
}

\begin{abstract}
Palabras clave: Microorganismos de montaña (MM); producción orgánica/ecológica; bioles; activaciones líquidas; insumos biológicos.

Keywords: Mountain microorganisms (MM); organic/ecological farming; liquid microbial cultures; liquid-phase fermentation; biological inputs.
\end{abstract}

Recibido: 22/04/2020

RESUMEN

Introducción. Los microorganismos de montaña (MM) son un insumo fundamental para la producción orgánica/ecológica en Costa Rica. Su uso genera preguntas relacionadas con el procedimiento para las activaciones líquidas, que presenten las mayores poblaciones microbianas. Objetivo. Evaluar factores clave que afectan la calidad de la fase líquida de MM, tales como el tiempo de activación, aireación, presencia de nutrientes, entre otros. Materiales y métodos. Se evaluaron factores asociados con las activaciones líquidas, tiempo de activación, disponibilidad de aireación, dosis de MM sólido, dosis de melaza y la adición de nutrientes inorgánicos solubles $(\mathrm{K})$ e insolubles $(\mathrm{P})$. Se determinaron poblaciones de bacterias, hongos filamentosos, bacterias fijadoras de nitrógeno (FN), microorganismos solubilizadores de fósforo (SP), lactobacilos (Lac) y levaduras (Lev), así como pH y $\mathrm{P}$ en solución. Los datos se sometieron a la prueba de Shapiro-Wilks. Las variables que no presentaron normalidad fueron evaluadas según

\footnotetext{
* Autora para correspondencia. Correo electrónico: leida.castro@ucr.ac.cr

1 Universidad de Costa Rica, Centro de Investigaciones Agronómicas-Agroalimentarias, San José, Costa Rica.
}

Aceptado: 22/06/2020

\section{ABSTRACT}

Factors related to liquid activation of Mountain Microorganisms (MM). Introduction. Mountain microorganisms (MM) are a fundamental input for organic/ecological farming in Costa Rica. Its use raises questions related to the procedure for liquid culture activation in order to attain the largest microbial populations. Objetive. To evaluate key factors that affect the quality of MM liquid phase culture, such as activation time, aeration, presence of nutrients, among others. Materials and methods. Factors associated with liquid-phase culture, incubation time, aeration availability, solid MM dosage, molasses dosage, and addition of soluble $(\mathrm{K})$ and insoluble $(\mathrm{P})$ inorganic nutrients were evaluated. Bacteria, filamentous fungi, nitrogenfixing bacteria (FN), phosphorus-solubilizing microorganisms (SP), lactobacilli (Lac), and yeast (Lev) populations were determined, as well as $\mathrm{pH}$ and phosphorus in solution. Data was subjected to the Shapiro-Wilks test. Variables that did not fit a normality test were evaluated according

(D) 0000-0002-3788-7173.

2 Departament of Plant Pathology and MicrobiologyIowa State University, EEUU. (D) 0000-0002-3215-8633. 
Kruskal Wallis y las normales con ANDEVA y LSD Fisher $(\mathrm{p}<0,05)$. Resultados y discusión. Las poblaciones cambiaron en el tiempo. El pH aumentó en el tiempo, mientras que los Lac y Lev disminuyeron. La disponibilidad de aireación redujo el tiempo de fermentación e incrementó las poblaciones, sin embargo, ocasionó un aumentó del pH en menor tiempo, por lo que los Lac y Lev disminuyeron mientras que las FN y SP incrementaron. La concentración más alta de melaza afectó negativamente la población de Lac, mientras que una reducción a la mitad en la cantidad de MM sólido, incrementó las poblaciones de SP y no afectó los otros grupos. La adición de una fuente orgánica de $\mathrm{K}$ aumentó las poblaciones, por tanto activados de MM reforzados pueden ser utilizados también como fuente de nutrientes. La adición de roca fosfórica no alteró las poblaciones, pero los datos sugieren que el fósforo solubilizado es retenido en la biomasa. Conclusión. Las condiciones de elaboración afectan la dinámica de las poblaciones microbianas presentes en las activaciones líquidas de MM.

\section{INTRODUCCIÓN}

El uso de insumos biológicos artesanales se ha popularizado entre las personas productoras agrícolas de Costa Rica y América Latina (Restrepo y Hensel 2015), sobre todo en la producción orgánica y ecológica. En Costa Rica estas personas producen, desde hace más de 20 años la totalidad de los insumos que utilizan entre los que se encuentran repelentes, biofermentos simples, biofermentos "reforzados" con hierbas, sales orgánicas o frutas, microorganismos de montaña, abonos orgánicos tipo compost, lombricompost y bocashi, entre otros. En países desarrollados está tendencia de uso de insumos biológicos es similar, en este caso, se utilizan especialmente formulaciones con microorganismos promotores de crecimiento vegetal (DíazZorita y Fernández-Canigia 2009). to the Kruskall-Wallis assessment method, and when they did fit normality were analyzed by ANOVA and Fisher's LSD test $(p<0.05)$. Results and discussion. Populations of functional microorganisms changed over time. $\mathrm{pH}$ increased during incubation, while Lac and Lev decreased. Increased air availability reduced fermentation time and increased microbial populations; however, this regime accelerated $\mathrm{pH}$ increase rate, along with faster decline of Lac and Lev, and increase of FN and SF. Higher molasses concentration negatively affected Lac populations, while halving the initial amount of solid MM resulted in increased SP populations and did not affect other microbial groups. Amending liquid culture with an organic source of $\mathrm{K}$ induced an increase in overall populations, therefore activated MM can also be used as a source of nutrients. Rock phosphate addition had no effect on microbial populations, but data suggests that solubilized phosphorus was fixed in microbial biomass. Conclusion. Production conditions affect the dynamics of microbial populations present in the liquid-phase activation of MM.

Particularmente, el uso de los microorganismos de montaña, tanto en la versión sólida como las activaciones líquidas (bioles de MM), se ha vuelto central y fundamental, gracias a las funciones que se les atribuye como inóculo microbiano, los cuales funcionan como mejorador de la biología del suelo, supresor de enfermedades, facilitador de la disponibilidad de elementos esenciales en el suelo, descomponedor de materia orgánica, optimizador en la producción pecuaria, controlador de malos olores, entre otros (Acosta 2012, Castro et al. 2015, Castro et al. 2020).

Las poblaciones microbianas benéficas presentes en los activados líquidos de MM se pueden clasificar principalmente en 4 grupos funcionales: fijadores de nitrógeno (FN), solubilizadores de fósforo (SP), lactobacilos (Lac) y levaduras (Lev) (Castro et al. 2015). Se ha determinado que al cuarto día de activación del 
MM se produce el crecimiento de principalmente hongos, a los 8 días bacterias y a los 15 días levaduras, sin embargo, en recuentos realizados en el Laboratorio de Microbiología del CIA-UCR, se ha observado la presencia de los 4 grupos funcionales antes mencionados, en altas proporciones en muestreos realizados en diferentes etapas después de la activación.

El rol de los microorganismos en el sistema suelo es clave para la productividad de los suelos y las plantas (Van Der Heijden et al. 2008). Los diferentes grupos funcionales presentes en los MM tienen potencial para colonizar el sistema radicular y/o la materia orgánica y establecerse activamente, al promover la síntesis de sustancias benéficas para las plantas, facilitar la absorción de nutrientes y fomentar la protección contra enfermedades, además, al solubilizar fosfatos y otros nutrientes esenciales, fijar $\mathrm{N}$, inducir la resistencia al estrés, estabilizar los agregados y mejorar la estructura del suelo (Castro et al. 2015, Hayat et al. 2010).

Los bioles de MM son insumos con una alta carga microbiológica, pero con bajos contenidos de nutrientes, por ello, condiciones como bajas cantidades de materia orgánica en el suelo, la utilización de agroquímicos y la mecanización pueden limitar las potencialidades de este inoculo microbiano. Mientras que un manejo adecuado de la incorporación de material orgánico y otras prácticas ecológicas, que brinden albergue y las condiciones idóneas para los microorganismos, son factores sinérgicos (Castro et al. 2015, Badilla 2019).

Los SP son de gran importancia para el crecimiento vegetal, ya que el $\mathrm{P}$, a pesar de que puede estar en grandes cantidades en los suelos, en la mayoría de los casos no se encuentra disponible para ser absorbido (Vargas-Barrantes y Castro-Barquero 2019). Los microorganismos, por medio de la solubilización y/o mineralización del fósforo inorgánico y orgánico, ponen a disposición de la planta el elemento. El principal mecanismo para la solubilización es la producción de ácidos orgánicos y la mineralización de $\mathrm{P}$ orgánico por medio de la acción enzimática
(Khan et al. 2009). Castro et al. (2015) mostraron que la utilización de activados líquidos de $\mathrm{MM}$ al suelo fueron capaces de incrementar las concentraciones de $\mathrm{P}$ en la solución del suelo.

Otros 2 grupos funcionales de importancia son los lactobacilos y las levaduras. Los primeros producen sustancias antimicrobianas supresoras de microorganismos con potencial fitopatogénico, además, de ser degradadores de materia orgánica. Asimismo, los segundos son importantes principalmente por su alta capacidad para degradar materia orgánica y por establecer relaciones simbióticas con las plantas a nivel del sistema radicular (Paul 1989, Acosta 2012, Pacheco y Uribe 2006).

Debido a que a nivel nacional el uso de MM se ha generalizado en la producción orgánica/ecológica, se ha generado interés también en la producción agropecuaria "convencional". De esta manera, personas productoras de leche y ganado, de la Zona Norte del país, lo emplean para eliminar el mal olor y optimizar la descomposición de las excretas de los animales y producir abono orgánico. Algunas personas productoras de pimienta y piña de la zona Caribe y Norte lo emplean para el manejo de enfermedades y mejorador de las propiedades del suelo. En la zona de Los Santos algunas fincas productoras de frutales de altura y leche producen sus MM para diversos fines y algunos de hortaliza, en el Valle Central, incorporan MM líquido vía fertirriego para controlar agentes patógenos y mejorar la salud del suelo y los cultivos. En la zona de Sarapiquí, fincas de piña orgánica han producido hasta 9000 L de MM activado por mes, el cual se ha aplicado semanalmente a toda la plantación.

Surgen, entonces, interrogantes sobre cuál es la mejor vía para la producción de este insumo. Por lo tanto, el objetivo de este trabajo fue evaluar los factores claves que influyen sobre las características del MM activado, conocido como fase líquida o bioles de MM. Para ello, se planteó evaluar a través del tiempo, el $\mathrm{pH}$ y las poblaciones de 4 de los grupos funcionales presentes en los activados de MM, elaborados 
con 2 diferentes mantillos. Además, determinar el efecto de la aireacción, la cantidad de melaza que es un material barato, por lo que se utiliza muchas veces en mayor cantidad con la idea de lograr un mejor producto, el tiempo de activación, la cantidad de MM sólido inicial, la adición de una fuente mineral soluble de potasio y una fuente poco soluble de fósforo (roca fosfórica).

\section{MATERIALES Y MÉTODOS}

Sitio experimental. Tanto los MM sólidos como las activaciones (MM líquido) se elaboraron en las instalaciones del Laboratorio de Microbiología de Suelos, CIA, UCR. En el mismo lugar se realizaron las evaluaciones biológicas y químicas pertinentes.

Variables evaluadas. Según correspondiera, se determinaron las poblaciones de microorganismos de los siguientes grupos: bacterias, hongos filamentosos, bacterias fijadoras de nitrógeno (FN), microorganismos solubilizadores de fósforo (SP), lactobacilos (Lac) y levaduras (Lev). Se llevó a cabo con diluciones seriadas y recuentos viables según las metodologías utilizadas en el Laboratorio de Microbiología Agrícola de la Universidad de Costa Rica (LMA-CIA 2015). Asimismo, se determinó cuando así se requirió, el $\mathrm{pH}$ y la conductividad eléctica (CE) con un potenciómetro-conductímetro portátil (Metter Toledo, Seven go duo) en el Laboratorio de Suelos y Foliares del CIA y el P en solución por medio de Espectroscopia de Emisión Óptica de Plasma Acoplado Inductivamente (ICP-OES). Las pruebas que requerían comparación de diferentes tratamientos contaron con 3 repeticiones.

Evaluación de los activados líquidos de MM preparado con mantillo de bosque de 2 sitios en diferentes tiempos de activación. Se confeccionó MM sólido y la activación líquida, según la metodología de Castro (2014) y Tencio (2015), se consideró como fuente, el mantillo de un bosque secundario de Río Frío y otro de Coronado, con el fin de observar si presentaban poblaciones similares. Para la elaboración de la versión sólida, se utilizaron recipientes de vidrio de 2 L con cierre hermético y salida de gases; para los bioles se utilizaron recipientes con las mismas características pero de $1 \mathrm{~L}$. Con el objetivo de evaluar los cambios microbiológicos en el tiempo, la activación líquida del MM de Río Frío fue evaluada por 136 días, en condiciones aeróbicas pero sin aire forzado, con muestreos por duplicado, los días $4,12,19,27,42,59,83,122$ y 136 días después de la activación (DDA). La activación líquida del MM procedente de Coronado se evaluó bajo las mismas condiciones por 40 días, con muestreos por duplicado, los días 5, 12, 26 y 40 DDA. Además, de evaluar las poblaciones, se determinó el pH. En el activado de Río Frío se evaluaron los siguientes grupos funcionales: Lac, Lev, hongos filamentosos y bacterias totales y en el de Coronado, y el resto de los ensayos las poblaciones de Lac, Lev, SF y los FN. Los siguientes ensayos se realizaron con MM proveniente de Coronado.

Disponibilidad de aireación en la activación. Se probaron 3 tipos de aireación con el fin de facilitar la disponibilidad de $\mathrm{O}_{2}$ para los microorganismos: con inyección de aire, durante todo el periodo de activación, por medio de un motor de pecera; aireacción pasiva, mediante un recipiente de vidrio sin cierre hermético y agitación esporádica para permitir el intercambio gaseoso ("50/50"); y sin aireacción, que correspondió a un recipiente de vidrio sin entrada de aire. Las evaluaciones se realizaron a los 12 DDA y se evaluaron los 4 grupos funcionales propuestos.

Debido a las diferencias observadas en las poblaciones de los grupos funcionales y el valor del $\mathrm{pH}$, según la disponibilidad de aireación, se evaluó, a los 5, 12, 20, 26 y 35 DDA, el efecto de la inyección de aire en el proceso de elaboración. Se utilizaron 3 repeticiones y se determinaron las poblaciones de grupos funcionales y el $\mathrm{pH}$.

Utilización de 2 dosis de inoculo de MM sólido. Para determinar si es factible reducir la cantidad utilizada de MM sólido en las activaciones líquidas, se confeccionó la fase líquida 
con la utilización de 2 dosis de MM sólido: $50 \%$ (20 g.L $\mathrm{L}^{-1}$ agua) y $100 \%$ (40 g.L - $^{-1}$ agua) de la dosis recomendada (Castro 2014, Tencio 2015, Castro et al. 2015). Las evaluaciones se realizaron a los 12 DDA y se evaluaron el $\mathrm{pH}$ y los 4 grupos funcionales (Lac, Lev, FN y SP).

Dosis de melaza. Se evaluaron 4 dosis de melaza: sin melaza (SM), la dosis recomendada por Castro (2014) y Tencio (2015) (1X: 19 mL.L-1 agua), 2 veces la dosis recomendada $(2 \mathrm{X})$ y 3 veces la dosis recomendada (3X). En este caso, se determinó a los 12 DDA como indicador, solamente la población de Lac y levaduras.

Enriquecimiento del proceso de activación con una fuente soluble de potasio. Para determinar el efecto de la aplicación de una fuente soluble de $\mathrm{K}$ sobre las poblaciones de los grupos funcionales evaluados (Lac, Lev, FN y SP), se agregó 3 DDA 10 gr. $\mathrm{L}^{-1}$ de $\mathrm{K}_{2} \mathrm{SO}_{4}$ de fuente mineral y se procedió a realizar la evaluación a los 12 DDA mediante la comparación con un testigo sin adición de la sal.

Aplicación de roca fosfórica en proceso de activación. Debido a la baja solubilidad de la roca fosfórica, comparada con las sales, se realizó un ensayo para evaluar el efecto en los activados de la adición de RF sobre el $\mathrm{pH}$, el $\mathrm{P}$ en solución y sobre las poblaciones de los grupos funcionales. El ensayo se llevó a cabo por medio de la inyección de aire forzado, que se agregó al activado 3 DDA 10 g.L - $^{-1}$ de RF con un tamaño de partícula entre 90 y $500 \mu \mathrm{m}$, y se evaluó a los 12 y 35 DDA. Los tratamientos evaluados fueron: Agua más $\mathrm{RF}\left(\mathrm{H}_{2} \mathrm{O}+\mathrm{RF}\right)$, activado de MM sin RF (MM), Activado de MM con RF (MM + RF), Activado de MM autoclavado y con RF (MMA + P). La adición de $\mathrm{RF}$, a los tratamientos que lo requerian, se realizó 3DDA. La RF fue autoclavada previamente, en forma sólida, en recipientes de vidrio.

Análisis estadístico. Los datos de los ensayos, donde se evaluaron diferentes tratamientos se sometieron a la prueba de ShapiroWilks (modificado) para evaluar la normalidad.
Aquellas variables que no presentaron normalidad fueron evaluadas según el análisis Kruskal Wallis, las variables normales según el ANDEVA y la prueba LSD Fisher $(\mathrm{p}<0,05)$ como separador de medias. Para algunos ensayos se muestra la desviación estándar de los datos, que permite una mejor comprensión.

\section{RESULTADOS Y DISCUSIÓN}

Respecto a la evaluación de los activados líquidos de MM a través del tiempo (Figura 1), para el activado de Rio Frío, ya para el día 42, el pH se incrementó más allá de los 4,5, hasta alcanzar un valor superior a 6 a los 136 días. Entre los 40 y los 136 días la población de lactobacilos disminuyó al pasar de un valor cercano a 8 ( $\log _{10}$ de las ufc) a un valor de 5. Las levaduras presentaron los mayores valores entre los 20 y los 40 días, mientras que las bacterias mostraron un crecimiento más errático, pues alcanzaron valores superiores a 8 e inferiores a 6 en diferentes momentos de muestreo. El activado de Coronado fue evaluado en las mismas condiciones hasta los 40 días cuando se observó incremento en el $\mathrm{pH}$, hasta alcanzar un valor mayor a 4,5 (Figura 1). En este caso se evaluaron las poblaciones de los grupos funcionales: lactobacilos, levaduras, solubilizadores de P y fijadores de $\mathrm{N}$, todas las poblaciones disminuyeron con el tiempo después de los 12 días. Bajo las condiciones de elaboración (aérobicas sin aire forzado) los bioles de MM pueden ser utilizados desde los primeros días, hasta los 40 días después de la elaboración, con su aporte microbiano mayor antes de los 25 días. Es importante destacar que se evaluaron también las poblaciones de hongos filamentosos, pero no se encontró presencia de los mismos en los activados, posiblemente debido a las condiciones semiaeróbicas en los líquidos y al muy bajo $\mathrm{pH}$ de los activados en los primeros días de la elaboración. En este sentido Badilla (2019) reportó la presencia del hongo filamentoso Mucor sp., pero con crecimiento aislado, siempre sobre la superficie de activados provenientes de mantillo de arroz. 

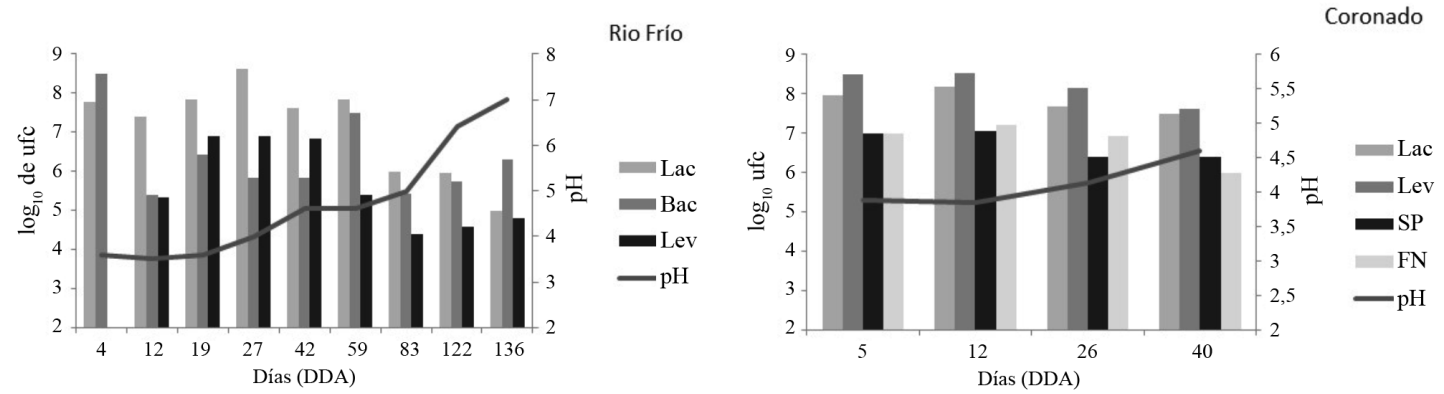

Figura 1. Poblaciones microbianas $\left(\log _{10}\right.$ de ufc. $\left.\mathrm{ml}^{-1}\right)$ de bacterias, lactobacilos, levaduras, solubilizadores de P, fijadores de $\mathrm{N} \mathrm{y} \mathrm{pH} \mathrm{en} \mathrm{las} \mathrm{activaciones} \mathrm{líquidas} \mathrm{de} \mathrm{MM} \mathrm{de} \mathrm{las} \mathrm{localidades} \mathrm{de} \mathrm{Río} \mathrm{Frío} \mathrm{y} \mathrm{Coronado.} \mathrm{Cada} \mathrm{dato} \mathrm{corresponde} \mathrm{al}$ promedio de 2 repeticiones.

Disponibilidad de oxígeno en la activación. Una interrogante frecuente cuando se realiza la activación de los $\mathrm{MM}$, es si se debe hacer de manera aeróbica o anaeróbica; actualmente algunas personas productoras los preparan en condiciones aerobias y otros en condiciones anaerobias. Según los resultados obtenidos (Figura 2), los bioles con inyección de aire presentaron las mayores poblaciones de microorganismos a los 12 DDA, sin embargo, el $\mathrm{pH}$ en este momento ya se encontraba alrededor de 4,5, mientras que en los tratamientos anaeróbico completo (tapa hermética para evitar entrada de aire) y aeróbico pasivo (sin inyección de aire), el pH mostró valores menores a 4 (Figura 2).
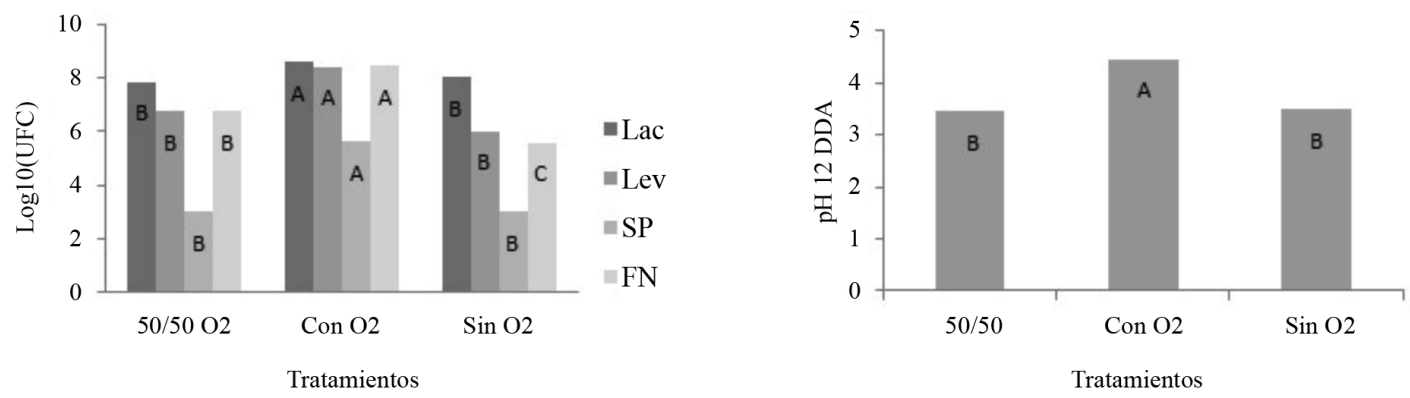

Figura 2. Efecto de la aireación sobre las poblaciones $\left(\log _{10}\right.$ de ufc.ml-1) de lactobacilos, levaduras, solubilizadores de P, fijadores de $\mathrm{N}$ y pH en el proceso de activación líquida de $\mathrm{MM}$ a los $12 \mathrm{DDA}$. Barras con diferente letra para el mismo grupo funcional corresponden a diferencias estadísticas significativas $(\mathrm{p}<0,05)$.

Al realizar una evaluación por un periodo de tiempo mayor, en condiciones con bombeo de aire (Figura 3), se observó que a los 12 días DDA el $\mathrm{pH}$ era ya de 5, a los 20 DDA superaba los 6 y a los 35 DDA sobrepasaba los 7. Este hecho se relacionó directamente con una reducción en las poblaciones de lactobacilos y levaduras, mientras que se observó que los FN y los SP mostraron las poblaciones más altas al inicio y al final de la activación. De esta manera, una activación en presencia de bombeo de aire, para asegurar el suministro de oxígeno, mostró un aumento en el $\mathrm{pH}$ más acentuado y en menor tiempo, con una reducción de lactobacilos y levaduras y un aumento en los SP, esto es importante de tomar en cuenta, según el interés que se tenga en el activado. 


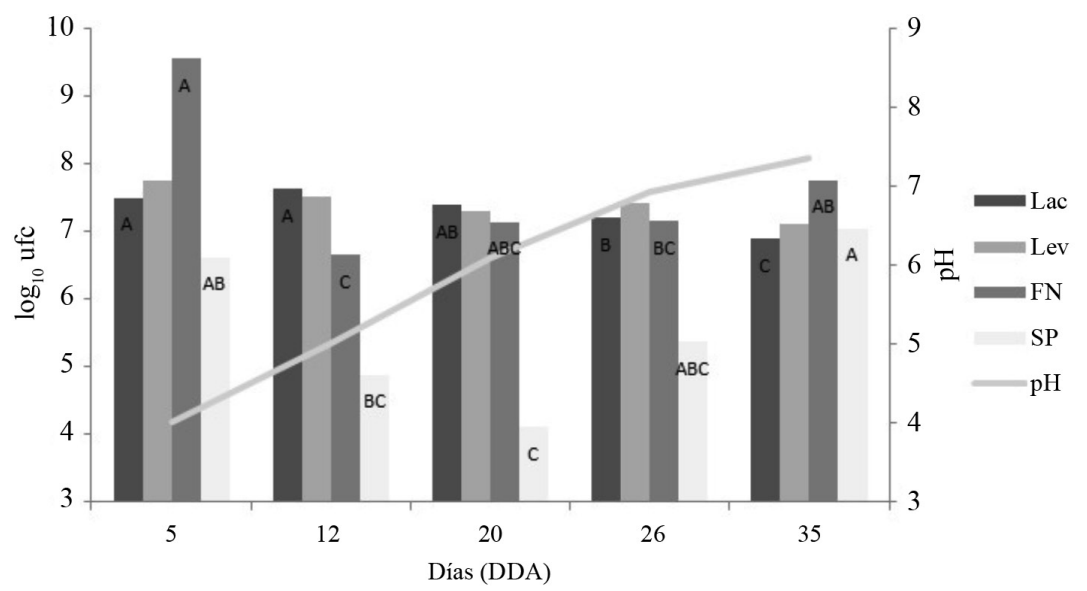

Figura 3. Poblaciones $\left(\log _{10}\right.$ de $u$ fc. $\left.\mathrm{ml}^{-1}\right)$ de lactobacilos, levaduras, solubilizadores de $\mathrm{P}$, fijadores de $\mathrm{N}$ y $\mathrm{pH}$ en las activaciones líquidas de MM con inyección de aire forzado, a través del tiempo. Barras con diferente letra para el mismo grupo funcional corresponden a diferencias estadísticas significativas $(\mathrm{p}<0,05)$.

Si el objetivo es la aplicación de la mayor cantidad de lactobacilos y levaduras, las aplicaciones se deben realizar alrededor de los 12 DDA, si el interés son los grupos funcionales FN y SP se pueden utilizar, al inicio de la activación para los $\mathrm{FN}$ o posterior a los 26 días para los SP. Una estrategia de aplicación más exitosa puede aprovechar todo el ciclo de producción del activado, con aplicaciones en todas las fases. Si la activación se realiza con inyección de aire forzado los sustratos que promueven el crecimiento de Lac y Lev en el activado se agotarán más rápidamente, así también se producirá un aumento en el $\mathrm{pH}$, mientras que si se mantiene aeróbico, de manera pasiva, esto es sin inyección de aire y sin tapa hermética, el pH tardará más tiempo en aumentar y el activado se podrá utilizar por un periodo mayor como fuente de Lac y Lev. Por otro lado, según los resultados obtenidos, la anaerobiosis completa afecta negativamente al menos las poblaciones de FN (Figura 2).

Dosis de inóculo de MM sólido para la activación líquida. Debido a que el MM sólido tiene como componente mantillo de bosque, la extracción del mismo puede convertirse en un factor negativo para el medio natural. Los insumos de este tipo han sido generados en producciones agrícolas ecológicas y no para la agricultura industrial, sin embargo, debido a que empíricamente ha mostrado tener un impacto positivo también en la agricultura convencional, es necesario crear estrategias que permitan un uso racional de este insumo.

En este sentido las personas autoras proponen que la legislación nacional contemple que cada proyecto productivo extensivo que implemente su uso, mantenga áreas destinadas a la conservación boscosa, desde donde se pueda hacer un uso racional de este recurso. Además es importante definir si es factible reducir la cantidad utilizada de MM sólido, por lo que se evaluó en este estudio si la utilización de la mitad de la cantidad de MM sólido utilizada regularmente (Castro 2014, Tencio 2015) tendría algún efecto sobre las poblaciones de los 4 grupos funcionales evaluados. Se observó que las poblaciones de Lac, Lev y FN fueron muy similares y que las poblaciones de los SP fueron mayores al utilizar la mitad de la cantidad de MM sólido (Figura 4). De ello, se deduce que se puede reducir la cantidad propuesta en Castro (2014) y Tencio (2015) a la mitad. 


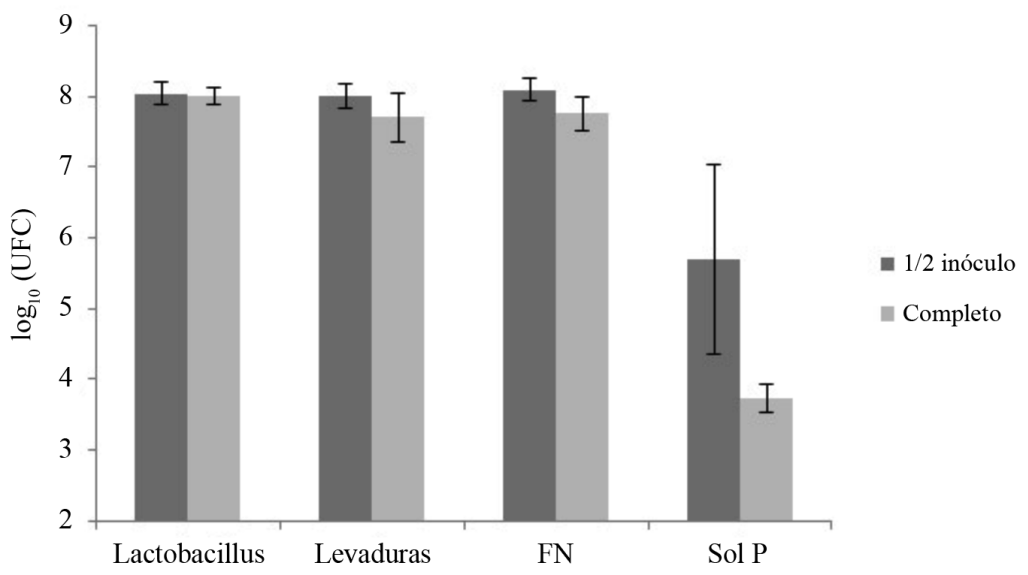

Figura 4. Poblaciones microbianas $\left(\log _{10}\right.$ de $\left.\mathrm{ufc} \cdot \mathrm{ml}^{-1}\right)$ de lactobacilos, levaduras, fijadores de $\mathrm{N}$ y solubilizadores de $\mathrm{P}$ en las activaciones líquidas de MM con 2 cantidades de inóculo sólido a los 12 DDA. Se muestran las barras para la desviación estándar.

Dosis de melaza. Al evaluar las poblaciones de Lev y Lac en diferentes dosis de melaza, se observó que las poblaciones de Lac presentaron diferencias significativas (Figura 5). El tratamiento $1 \mathrm{x}$ (dosis recomendada) permitió el mayor crecimiento de este grupo funcional microbiano.
Es importante, por tanto, no excederse en la cantidad de melaza utilizada para las activaciones, ya que su composición puede aumentar la CE del medio (Fajardo y Sarmiento 2007), como se observó para el presente ensayo (Tabla 1) y afectar algunas poblaciones microbianas (Yana et al. 2015).

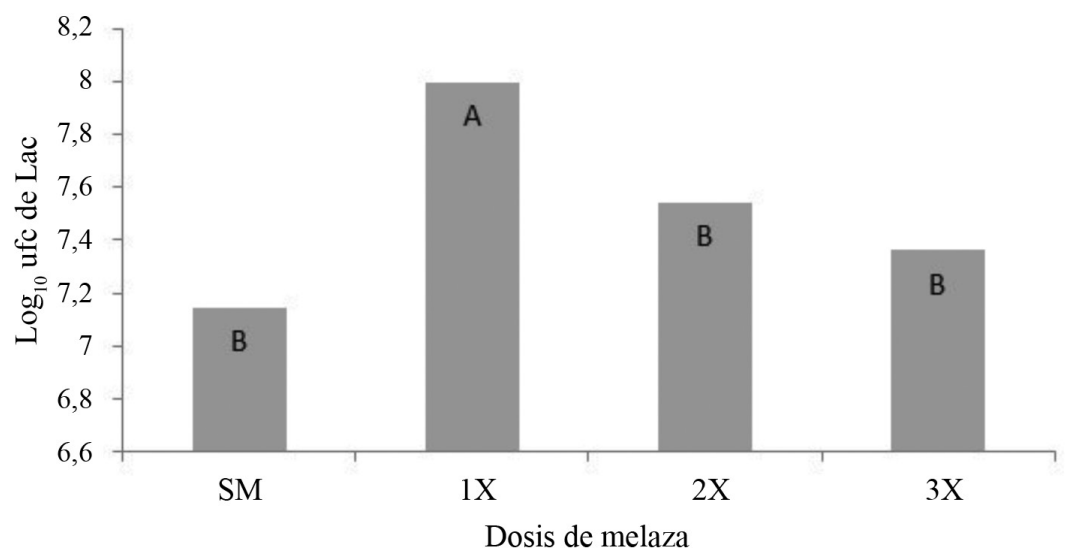

Figura 5. Población de $\operatorname{Lac}\left(\log _{10}\right.$ de ufc. $\left.\mathrm{ml}^{-1}\right)$ según 3 diferentes dosis de melaza utilizada en la activación líquida a los 12 DDA. Barras con diferente letra para el mismo grupo funcional corresponden a diferencias estadísticas significativas $(\mathrm{p}<0,05)$. 
Tabla 1. Valores de $\mathrm{pH}$ y conductividad eléctrica (CE) según tratamientos evaluados bajo 4 dosis de melaza a los 12 DDA.

\begin{tabular}{ccc}
\hline Tratamiento & $\mathrm{pH}$ & $\mathrm{CE}\left(\mathrm{mS} \cdot \mathrm{m}^{-1}\right)$ \\
\hline $\mathrm{SM}$ & $4,87 \mathrm{a}$ & $1,82 \mathrm{~d}$ \\
$1 \mathrm{X}$ & $4,71 \mathrm{ab}$ & $4,48 \mathrm{c}$ \\
$2 \mathrm{X}$ & $4,19 \mathrm{~b}$ & $6,58 \mathrm{~b}$ \\
$3 \mathrm{X}$ & $4,18 \mathrm{~b}$ & $9,35 \mathrm{a}$ \\
\hline
\end{tabular}

Enriquecimiento del proceso de activación con una fuente soluble de potasio. Una práctica que se ha extendido es el enriquecimiento de los activados líquidos de MM con sales de fuente natural de diversos nutrientes como $\mathrm{K}$, $\mathrm{Ca}, \mathrm{Mg} \mathrm{u}$ otros elementos menores como Mn y B. En este caso, se determinó el efecto del enriquecimiento con $\mathrm{K}\left(\mathrm{K}_{2} \mathrm{SO}_{4}\right)$ sobre los 4 grupos funcionales determinados; se encontró que a los 12 DDA todas las poblaciones aumentaron por el enriquecimiento con la sal (Figura 6). Esta observación concuerda con las realizadas previamente en un estudio ejecutado en el Laboratorio de Microbiología Agrícola-UCR, donde Vega (2017) encontró que el enriquecimiento de los activados líquidos de $\mathrm{MM}$, con soluciones de $\mathrm{K}, \mathrm{Ca}, \mathrm{Mg}, \mathrm{S}, \mathrm{B}, \mathrm{Cu}, \mathrm{Fe} \mathrm{Mg}$ y Zn, incrementó en todos los casos las poblaciones Lac, Lev, FN y SP hasta en el orden de 10 y 100 veces, según el grupo funcional evaluado. La utilización de los activados de MM enriquecidos con sales, representa un aporte no solo de inóculo microbiano en mayor concentración, sino también de nutrientes. Por otro lado, Aguado-Santacruz et al. (2012) indican que los microorganismos pueden promover el crecimiento vegetal a traves de la produccion de sustancias quelantes, como es el caso de los sideróforos.

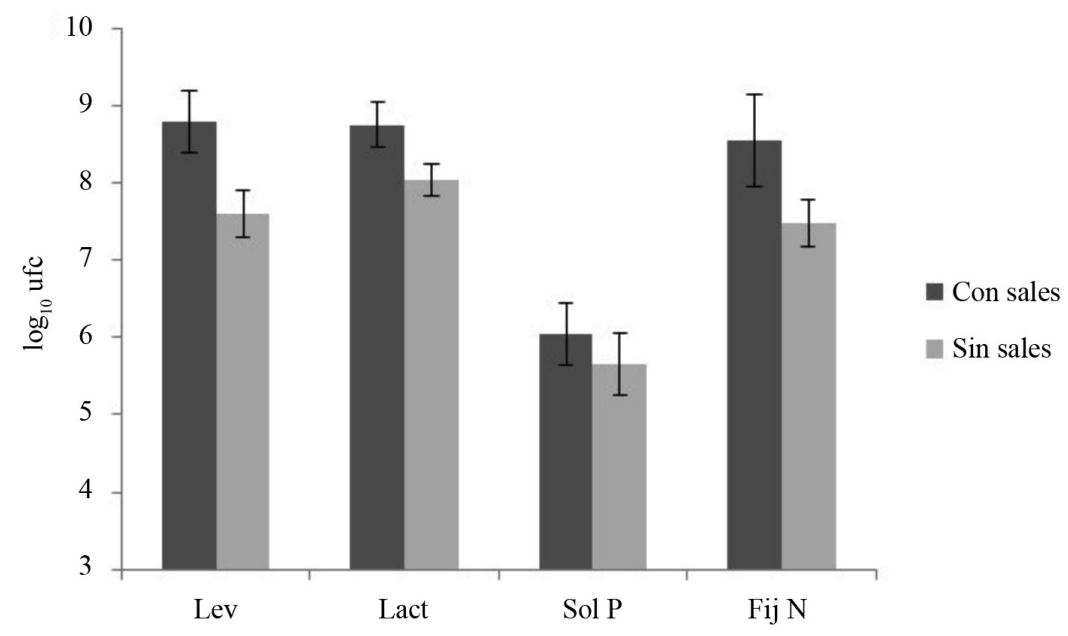

Figura 6. Poblaciones microbianas $\left(\log _{10}\right.$ de $\left.u f c \cdot \mathrm{ml}^{-1}\right)$ de lactobacilos, levaduras, fijadores de $\mathrm{N}$ y solubilizadores de $\mathrm{P}$ en las activaciones líquidas de $\mathrm{MM}$ con la adición de sales con potasio $\left(\mathrm{K}_{2} \mathrm{SO}_{4}\right)$ de fuente mineral. Se muestran las barras de error.

Enriquecimiento del proceso de activación con una fuente poco soluble de fósforo. La solubilidad de la roca fosfórica en agua, se mantuvo baja durante todo el periodo de experimentación, ya que fue menor de $0,24 \mathrm{mg} \cdot \mathrm{L}^{-1} \mathrm{a}$ los 12 y los 35 DDA (Figura 7). A los 12 DDA la cantidad de $\mathrm{P}$, en solución, fue muy similar entre el activado con RF y el que no tenía, sin embargo, al someter el activado al autoclavado para eliminar los microorganismos, se observó que la 
cantidad de $\mathrm{P}$ en solución tendió a aumentar. Para la evaluación 35 DDA, se incrementó ligeramente la cantidad de $\mathrm{P}$ en solución en el tratamiento MM, pero la cantidad de $\mathrm{P}$ se redujo en el tratamiento MMP, mientras que el tratamiento autoclavado presentó la cantidad mayor de $\mathrm{P}$ en solución. Lo anterior se explica al menos por las características ácidas del producto que solubiliza el $\mathrm{P}$ de la RF, pero en presencia de microorganismos este $\mathrm{P}$ es retirado como forma soluble y/o retenido en la biomasa, lo que concuerda con el aumento en las poblaciones de SP y FN entre la primera y segunda evaluación, tanto en el activado con P como el que no se adicionó con P (Figura 8).

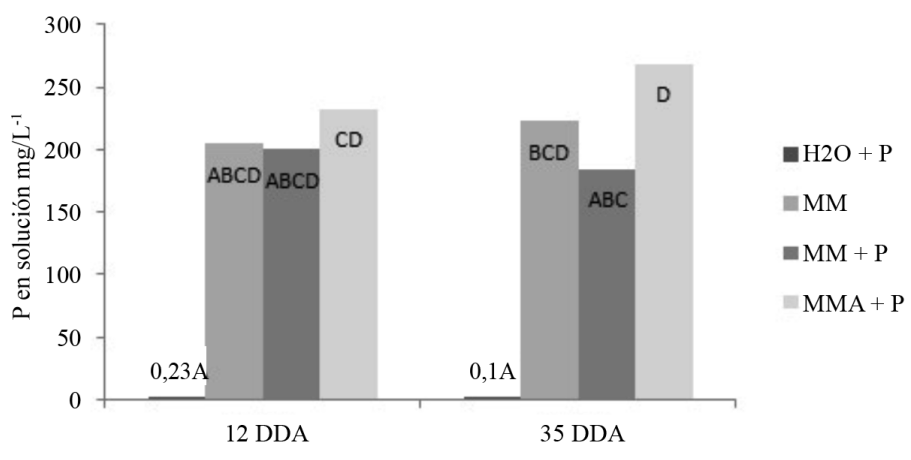

Figura 7. Cantidad de P en solución en mg.L $\mathrm{L}^{-1}$ a los 12 y 35 DDA en los diferentes tratamientos. Barras con diferente letra para el mismo grupo funcional corresponden a diferencias estadísticas significativas $(\mathrm{p}<0,05)$.

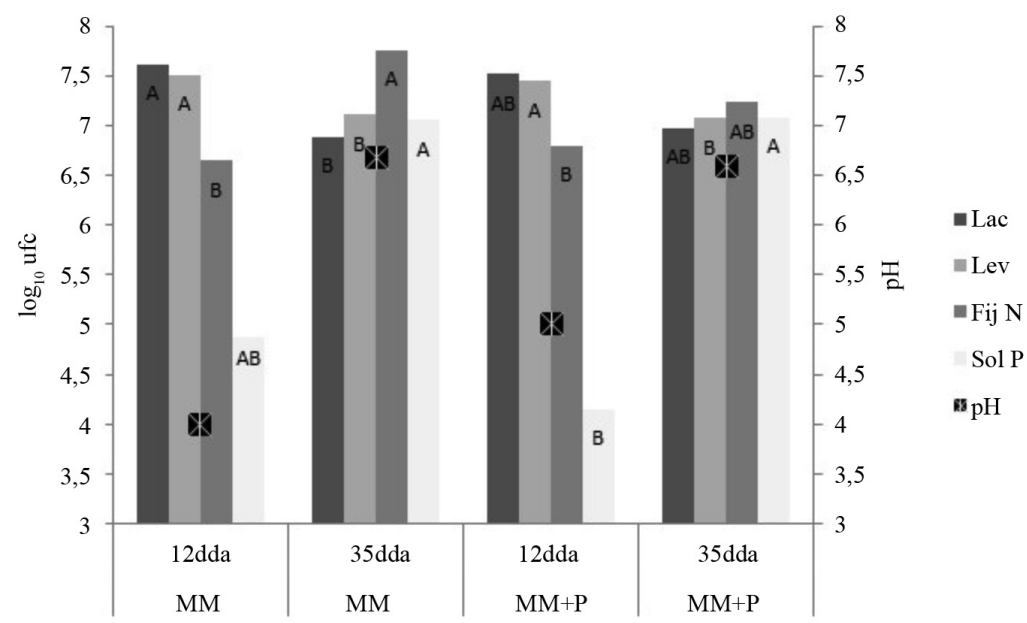

Figura 8. Poblaciones microbianas $\left(\log _{10}\right.$ de ufc. $\left.\mathrm{ml}^{-1}\right)$ de lactobacilos, levaduras, fijadores de $\mathrm{N}$ y solubilizadores de $\mathrm{P}$ y $\mathrm{pH}$ en las activaciones líquidas de $\mathrm{MM}$ con la adición de roca fosfórica $(\mathrm{P})$. Barras con diferente letra para el mismo grupo funcional corresponden a diferencias estadísticas significativas $(\mathrm{p}<0,05)$.

Como ya se discutió anteriormente, en este caso, las poblaciones de Lac y Lev también se redujeron con el tiempo, mientras que el pH aumentó (Figura 8 y Figura 9). Para los tratamientos $\mathrm{H}_{2} \mathrm{O}+\mathrm{P}$ y MMA $+\mathrm{P}$ los valores de $\mathrm{pH}$ se mantuvieron constantes, para el primero alrededor de 7 y para el segundo a 4 . $\mathrm{La}$ adición de RF no afectó a las poblaciones de los grupos funcionales evaluados (Figura 8), pero los datos sugieren que el fósforo solubilizado de 
la RF es incorporado en la biomasa microbiana o trasformado a formas no solubles (Figura 7). En este sentido, $\mathrm{Xu}$ et al. (2020) indican que la inmobilización microbiana de $\mathrm{P}$ reduce las formas solubles, que son en condiciones de campo susceptibles a la lixiviación.

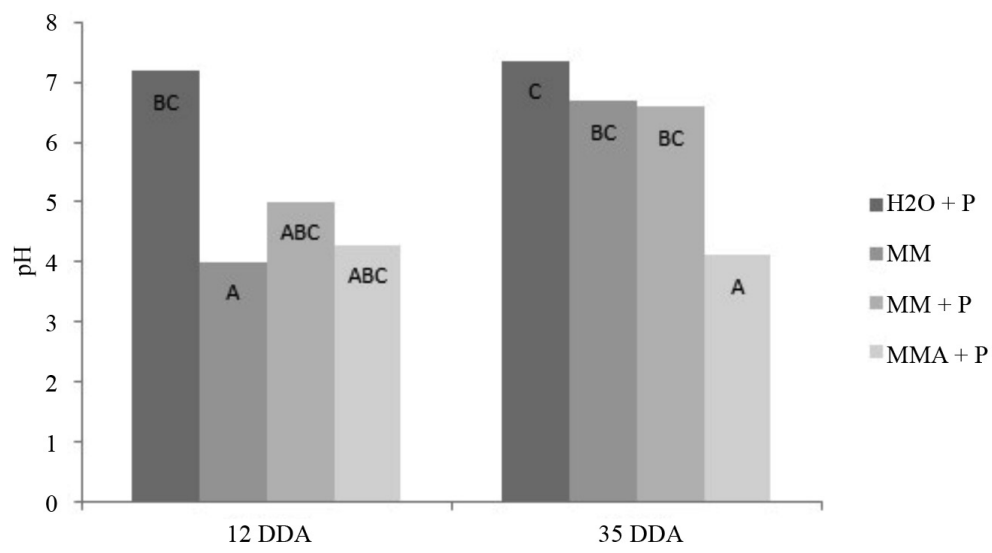

Figura 9. Valor del pH a los 12 y 35 DDA en los diferentes tratamientos con RF (P) añadida. Barras con diferente letra para el mismo grupo funcional corresponden a diferencias estadísticas significativas $(p<0,05)$.

\section{CONCLUSIÓN}

Los activados de MM representan una fuente importante de inóculo microbiano con potencial para el uso agropecuario. Las cargas microbiológicas de los grupos evaluados oscilan entre $10^{6}$ y $10^{8}$, según el tiempo trascurrido desde la activación, como de las condiciones en el proceso de elaboración. De esta manera, según se observó, las poblaciones y el pH se ven influenciados por la utilización de aire forzado en las activaciones. Dichos activados pueden también utilizarse para la incorporación de nutrientes al sistema, con la ventaja de que puede inducir la quelatación para una mejor absorción por la planta.

Se recomienda el uso de 20-25 g de MM sólido. $L^{-1}$ agua para realizar la activación, asimismo se insta a investigar si dosis más bajas puedan ser empleadas sin que las poblaciones microbianas sufran reducciones significativas. Otro aspecto a considerar es validar cuántas activaciones puede soportar un mismo inóculo de MM sólido inicial, sin que su aporte de inóculo microbiano al activado, disminuya de forma significativa.

Además, las diferencias observadas, en los preparados con y sin aire forzado, permitirán diseñar estrategias para programar las aplicaciones, según el efecto deseado en el sistema de producción, ya sea para solventar necesidades en cuanto a nutrición, fitosanidad, descomposición de residuos $\mathrm{u}$ otros factores relacionados a los grupos funcionales.

\section{LITERATURA CITADA}

Acosta, HA. 2012. Microorganismos eficientes de montaña: evaluación de su potencial bajo manejo agroecológico de tomate en Costa Rica. Effective local soil microorganisms. Turrialba, Costa Rica, CATIE. $100 \mathrm{p}$.

Aguado-Santacruz, G; Moreno-Gómez, B; JiménezFrancisco, B; García-Moya, E; Preciado-Ortiz, R. 2012. Impacto de los sideróforos microbianos y fitosideróforos en la asimilación de hierro por las plantas: una síntesis. Rev. Fitotec. Mex. 35(1):9-21. 
Badilla, S. 2019. Efecto de fermentos microbianos sobre el desarrollo de plántulas de caña de azucar (Saccharum officinarum L). Tesis Lic. Universidad de Costa Rica, Costa Rica. 66 p.

Castro, L. 2014. ¿Cómo hacer Microorganismos de Montaña $(\mathrm{MM})$ ?. Receta para Productores. Brochure. Costa Rica, PNAO-FITTACORI-UCR. 6 p.

Castro, L; Martínez, V; Castro, O; Blanco, M. 2020. Abono Orgánico, Microorganismos de Montaña (MM) y Fertibiol para el control biológico de la hernia de las crucíferas (Plasmodiophora brassicae wor.) en el cultivo de mostaza china (Brassica rapa $\mathrm{sp}$. pekinensis var. Taranko F1). Agronomía Costarricense 44(2):31-49.

Castro, L; Murillo, M; Uribe, L; Mata, R. 2015. Inoculación al suelo con Pseudomonas fluorescens, Azospirillum oryzae, Bacillus subtilis y Microorganismos de Montaña (MM) y su efecto sobre un sistema de rotación soya-tomate bajo condiciones de invernadero. Agronomía Costarricense 39(3):21-36.

Díaz-Zorita, M; Fernández-Canigia, MV. 2009. Field performance of a liquid formulation of Azospirillum brasilense on dryland wheat productivity. European Journal of Soil Biology 45(1):3-11.

Fajardo, EE; Sarmiento, SC. 2007. Evaluación de la melaza de caña como sustrato para la producción de Saccharomyces cerevisiae. Tesis de Grado. Pontificia Universidad Javeriana, Bogotá, Colombia. 120 p.

Hayat, R; Ali, S; Amara, U; Khalid, R; Ahmed, I. 2010. Soil beneficial bacteria and their role in plant growth promotion: a review. Annals of Microbiology 60(4):579-598.

Khan, AA; Jilani, G; Akhtar, MS; Saqlan, SM; Rasheed, M. 2009. Phosphorus Solubilizing Bacteria: Occurrence, Mechanisms and their Role in Crop Production. Journal of Agriculture and Biological Sciences 1(1):48-58.
LMA-CIA (Laboratorio de Microbiología AgrícolaCentro de Investigaciones Agronómicas). 2015. Metodología propuesta para la evaluación de microorganismos. Universidad de Costa Rica, San José, Costa Rica. 47 p.

Pacheco, F; Uribe, L. 2006. Lactofermento: Una alternativa en la producción de abonos orgánicos líquidos fermentados. Cartago, Costa Rica, INA. 18 p.

Paul, EA. 1989. Soil microbiology and biochemistry. 3 ed. Burlington, Massachusetts, USA, Elsevier. 514 p.

Restrepo, J; Hensel, J. 2015. El ABC de la agricultura orgánica, fosfítos y panes de piedra. Cali, Colombia, CJairo Restrepo Rivera. 397 p.

Tencio, R. 2015. Guía de elaboración y aplicación de bioinsumos para una producción agrícola más sustentable. San José, Costa Rica, PNAO-MAG. 14 p.

Van Der Heijden, MG; Bardgett, RD; Van Straalen, NM. 2008. The unseen majority: soil microbes as drivers of plant diversity and productivity in terrestrial ecosystems. Ecology letters 11(3):296-310.

Vargas-Barrantes, P; Castro-Barquero, L. 2019. Aislamiento y evaluación se microorganismos solubilizadores de fósforo de Andisoles de Costa Rica. Agronomía Costarricense 43(1):47-68.

Vega, A. 2017. Curvas de absorción de nutrientes y fertilización de almácigos orgánicos de papaya (Carica papaya) híbrido Pococí, Alajuela, Costa Rica. Tesis Lic. Universidad de Costa Rica, San José, Costa Rica. 54 p.

Xu, Z; Qu, M; Liu, S; Duan, Y; Wang, X; Brown, L; George, T; Zhang, L; Feng, G. 2020. Carbon addition reduces labile soil phosphorus by increasing microbial biomass phosphorus in intensive agricultural systems. Soil Use Management 36:536-546.

Yana, N; Marschnerc, P; Caoa, W; Zuoa, C; Qina, W. 2015. Influence of salinity and water content on soil microorganisms. International soil and water conservation Research 3:316-323. 